\title{
Barreras al uso de las Tecnologías de la Información y de la Comunicación en la enseñanza de Lenguas
}

\author{
Santiago Javier Galán \\ Universidade Federal do Amazonas \\ Wagner Barros Teixeira \\ Universidade Federal do Amazonas
}

\begin{abstract}
Resumo
O presente artigo busca traçar um paralelo sobre o uso das Tecnologias da Informação e da Comunicação em aulas de ensino de línguas entre as realidades das Regiões Norte e Sudeste do Brasil. Para tanto, constrói-se uma arena de vozes com base no(s) discurso(s) teórico de investigadores que tratam dessa temática e na experiência docente e científico-acadêmica dos autores. Após a reunião dessas vozes, conclui-se que, na realidade das Regiões brasileiras pesquisadas, o fator tempo é uma das principais barreiras a ser superada no tocante ao uso das TIC na prática em sala de aula, bem como à formação continuada docente.

Palavras-chave: Tecnologias da Informação e da Comunicação; ensino de línguas; tempo
\end{abstract}

\begin{abstract}
This paper compares the use of Communication and Information Technologies (CIT) in the contexts of language teaching classes in the regions of the North and Southeast of Brazil. To this end, a complex of voices is constructed, based on theoretical discourse(s) and on the teaching, scientific, and academic experience of the authors of this paper. From the analysis of these voices, it can be concluded that in the Brazilian regions studied the factor of time is one of the main barriers that need to be overcome concerning the use of CIT in classroom practices and in ongoing teacher development.
\end{abstract}

Keywords: Communication and Information Technologies; language teaching; time

\section{INTRODUCCIÓN}

El proceso de enseñanza-aprendizaje de una lengua, sea Materna, Segunda o Extranjera, está presente hace bastante tiempo en cuestionamientos y estudios de investigadores, teóricos y docentes involucrados con la temática. Durante el avance diacrónico de los estudios e investigaciones, para vencer las diferentes barreras y 
percances verificados, muchas veces, sincrónicamente, diferentes propuestas de trabajo se pensaron, se desarrollaron y se colocaron en práctica, con base en variadas corrientes teóricas, con el objetivo, muchas veces, de aplicación en clase. Tales dificultades afectan las diferentes instancias involucradas en el proceso: los discentes, las instituciones, los investigadores, entre otros; no obstante, nuestro foco aquí se reduce a algunas de aquéllas que enfrentamos, los docentes, eternos investigadores, quienes tratamos con la teoría, la enseñanza y la práctica de un idioma, principalmente a aquéllas que abordan el uso de las Tecnologías de la Información y de la Comunicación ${ }^{1}$ en clase.

Utilizamos la primera persona del plural no por motivos de gentileza o normativa académico-científica, sino por la esencia del diálogo que está presente en la composición de este trabajo. De modo que ese artículo posee un amplio bies dialógico de estudio e investigación en la acepción bajtiniana del término, pues, además de utilizar discursos teóricos de variados estudiosos e investigadores que abordan la temática, es el resultado del diálogo entre las indagaciones que realizamos en nuestros estudios de Especialización académica, con una mirada, principalmente, a las voces que forman el discurso de diferentes comunidades docentes brasileñas, más específicamente las de las Regiones Norte y Sureste del país.

Para fomentar más ese diálogo, enseguida, presentamos algunas consideraciones teóricas, oriundas de las voces de diferentes investigadores, con el objetivo de abrirle(s) espacio a la(s) voz(ces) del lector, quien, desde ahora, pasa a ponerse la máscara de coconstructor o co-autor de nuestro texto.

Como campo teórico, en primer lugar, describimos un breve panorama del proceso de enseñanza de lenguas en Brasil, dándoles especial atención a las dificultades verificadas durante ese proceso, en especial a aquéllas que son de la práctica docente y el uso de las TIC en las clases. Enseguida, hacemos un rápido viaje por la historia del uso de las TIC en clases de Lengua Extranjera ${ }^{2}$. También nos detenemos en una práctica ampliamente realizada por los docentes en su trabajo diario de formación; así, proponemos un rápido estudio sobre $\mathrm{la}(\mathrm{s})$ concepción(es) que se tiene de la exposición en clase.

Después vamos a compartir nuestra experiencia como profesores-investigadores, dándoles voces a las investigaciones que realizamos durante nuestros Cursos de

\footnotetext{
${ }^{1}$ En adelante: TIC.

${ }^{2}$ En adelante: LE
} 
Especialización. En ese momento, describimos cómo aconteció cada uno de esos trabajos y, enseguida, hacemos un breve análisis de los datos obtenidos, según nuestro objetivo principal que es el de trazar un paralelo entre las realidades del uso de las TIC en clases en el Norte y en el Sureste brasileños, más específicamente en los Estados de Amazonas y de Río de Janeiro.

Al final, con el objetivo de fomentar más ese diálogo, levantamos algunos cuestionamientos que consideramos pertinentes al estudio realizado, a fin de encaminarles la palabra a futuras voces, oriundas de nuevas investigaciones sobre el asunto.

\section{CONTRIBUCIONES DE VOCES DE OTROS INVESTIGADORES}

[...] Todo y cualquier texto posee una multiplicidad de significaciones, por eso no es posible considerar una única lectura como verdadera; el interlocutor hará aquélla que esté más de acuerdo con las "intenciones del texto" que se hacen presentes por medio de marcas lingüísticas qua van a funcionar como pistas que permitirán percibir el sentido global del texto (FÁVERO, 2000:56). ${ }^{3}$

Con base en la afirmación anterior, empleamos discursos teóricos que consideramos relevantes para la producción de nuestro trabajo, esperando, incluso, que le sirvan de estímulo al lector para que pueda (co)construir su(s) discurso(s) sobre la temática en discusión.

\section{La Lengua Extranjera en el panorama del proceso de enseñanza brasileña}

En Brasil, la enseñanza de Lenguas, en especial la del Español como Lengua Extranjera $^{4}$, está en evidencia en los últimos tiempos, principalmente a partir de la obligación de ofrecer esa lengua neolatina en la Secundaria en todas las esferas de la educación, a partir de la ley $11.161 / 2005^{5}$, lo que, teóricamente, aumenta la oferta en los cursos de graduación, y, por extensión, de pos graduación, con miras a satisfacer la demanda de profesionales graduados para la enseñanza de esa lengua, quienes cada vez más necesitan actualizar sus estudios. En medio de esa realidad, se encuentran los postulados de los PCN99 (BRASIL, 1999:26), que sugieren que la enseñanza de LE

\footnotetext{
${ }^{3}$ Original: [...] todo e qualquer texto possui uma multiplicidade de significações, não sendo possível considerar-se uma única leitura como verdadeira; o interlocutor fará aquela que estiver mais de acordo com as "intenções do texto" que se fazem presentes através de marcas lingüísticas que vão funcionar como pistas que permitirão perceber o sentido global do texto.

${ }^{4}$ En adelante: E/LE.

${ }^{5}$ Para más informaciones, vide la ley $11.161 / 2005$ en la íntegra a partir del link en las referencias.
} 
deje de objetivar

[...] solamente el conocimiento metalingüístico y el dominio consciente de reglas gramaticales [...]. Lo que da lugar, en la perspectiva actual, a una modalidad de curso que tiene como principio general llevar el alumno a comunicarse de modo adecuado en distintas situaciones de la vida cotidiana. ${ }^{6}$

Tal postura rescata el papel formador que la ley brasileña le otorgó a la Secundaria, lo cual había sido transferido tácitamente, durante décadas de enseñanza pautada en abordajes tradicionales y estructuralistas, a los institutos especializados en la enseñanza de Lenguas Extranjeras. Esos abordajes han estado de tal manera arraigados en la formación teórica de los docentes que se mantienen hasta el día de hoy en las clases brasileñas.

Sin embargo, para que el proceso de enseñanza-aprendizaje ocurra de forma significativa, es necesario que acontezca un cambio de actitud por parte de los docentes, quienes deben estar conscientes de su papel, así como de las dificultades propias del proceso, como la de la interrelación de la LE con las demás asignaturas del currículo escolar, que en la mayor parte, todavía se encuentra pautado en los modelos fragmentados, en contra del trabajo por áreas de conocimiento (PCN99, BRASIL, 1999). En vez de basar su trabajo en abordajes que, tradicionalmente, privilegian la enseñanza puramente estructural, es necesario que se busque aliar la enseñanza de las habilidades lingüísticas a las competencias sociolingüísticas, discursiva y estratégica, que involucran el proceso de adquisición de una LE, para poder amenizar el efecto nocivo de años de fosilización oriundo del trabajo docente estático, mecánico y repetitivo, utilizando para eso todos los recursos disponibles, sean teóricos, didácticos, tecnológicos y/o mediáticos.

A pesar de tal evidencia, además de los problemas mencionados, verificamos a partir de los PCN98 que desde la Primaria ni siempre “[...] la enseñanza de lengua extranjera [...] se ve como elemento importante en la formación del alumno, como un derecho que le debe ser garantizado (BRASIL, 1998:24)",7, puesto que, de acuerdo con las informaciones encontradas, a menudo la LE no consta en la base común de los currículos elaborados por las escuelas, recibiendo el rótulo de una actividad extra y

\footnotetext{
${ }^{6}$ Original: [...] apenas o conhecimento metalinguístico e o domínio consciente de regras gramaticais [...]. Cede lugar, na perspectiva atual, a uma modalidade de curso que tem como princípio geral levar o aluno a comunicar-se de maneira adequada em diferentes situações da vida cotidiana.

${ }^{7}$ Original: "[...] o ensino de Língua Estrangeira [...] é visto como elemento importante na formação do aluno, como um direito que lhe deve ser assegurado".
} 
sencilla, sin carácter promocional, siendo también dictada fuera del horario regular o incluso fuera de la escuela, por medio de convenios con instituciones de enseñanza que dictan cursos libres de idiomas, lo que la coloca fuera del contexto educacional escolar, en el cual está insertado el alumno.

Existen casos extremos, en los cuales, por falta de profesor calificado u otros motivos, la escuela no ofrece la LE en su matriz curricular o no la ofrece en algunas de los años, de forma seguida, perjudicando la continuidad del proceso de enseñanzaaprendizaje.

Además de los problemas institucionales verificados anteriormente, existen otros, con relación a las circunstancias de enseñanza y aprendizaje de la LE. En ese sentido, a partir de las informaciones encontradas en los PCN98, se verifican varias dificultades, entre ellas, la falta de material didáctico adecuado a la demanda de cada institución de enseñanza, considerando su cuerpo discente y su comunidad escolar, incluyendo aquí recursos tecnológicos y mediáticos; la ocurrencia de salones abarrotados, o sea, un ambiente con exceso de alumnos, dificultando la concentración, la orientación, el acompañamiento y, consecuentemente, el desarrollo de todo el proceso; el tiempo reducido dedicado a la enseñanza de LE en el currículo; así como profesores desestimulados, desactualizados y con falta de preparación para el ejercicio de su profesión, ya que faltan políticas de mejores salarios e inversión en la formación continua del docente.

A esos problemas, se puede añadir una dificultad de orden sociolingüístico, puesto que, en Brasil, país monolingüe de gran extensión territorial, salvo en algunos casos de comunidades inmigrantes, de poblaciones limítrofes con países hispanohablantes y de grandes metrópolis y ciudades de actuación turística como San Pablo, Río de Janeiro, Recife, Fortaleza, entre otras, la LE, exceptuando los casos de uso digital, vía Internet, no posee practicidad, valor funcional, para la mayoría de la población, pues no se usa en su cotidiano, o sea, no existe un contexto real de comunicación, de interacción discursiva, en el que se exija el uso de habilidades lingüísticas, principalmente de aquéllas destinadas a la producción y comprensión orales.

Por eso, la enseñanza de LE se direcciona casi que exclusivamente a la producción y a la comprensión escritas. Su base está en las habilidades de lectura y producción de textos, en vista de que poseen mayor practicidad para la población brasileña. 
En medio a tantos problemas y dificultades verificados durante el proceso de enseñanza de LE en nuestro país, basados en la legislación educacional vigente, encontramos la orientación para un trabajo continuo de construcción de conocimiento y de formación desde la Primaria, ya que a partir de esa etapa escolar,

[...] el alumno ya ha aprendido usos de lenguaje con los que tal vez no se haya familiarizado en casa [...], habiendo también interiorizado su naturaleza sociointeraccional [...] y empezado a construir conocimiento de naturaleza metalingüística en las clases de lengua materna. [...] Eso le posibilita pensar, hablar, leer y escribir sobre su propia lengua. En suma, el alumno ya sabe mucho sobre su lengua materna y sobre como usarla [...] (PCN98, BRASIL, 1998:28). ${ }^{8}$

Según tales orientaciones, considerando que el alumno de Primaria ya tenga conocimiento suficiente sobre su Lengua Materna, la LE debería estar presente en la formación del alumnado desde temprano. Sin embargo, conforme Moita Lopes (2001) afirma, eso ni siempre ocurre y la cuestión es más crítica en el contexto de la escuela pública brasileña. El lingüista evidencia y desmitifica la afirmación popular de que la clase social menos favorecida, principal público de esas escuelas, no posee aptitud para aprender una LE.

El investigador afirma que no existe evidencia lingüística que confirme tal afirmación. Se trata solamente de ideología, pues la enseñanza brasileña está pautada en los intereses y necesidades de la clase dominante, minoría que le otorga una justificativa social a la enseñanza de LE en la escuela, con predominio del Inglés, por influencia neo imperialista norte-americana, en vista de que, casi que exclusivamente, ésa es la parcela de la sociedad que, en la mayoría de las situaciones de las regiones de Brasil, excepto los casos ya mencionados, tendrá posibilidades de comunicarse en situaciones discursivas reales, dentro y fuera del país.

\section{Enseñanza de lenguas y TIC: voces que hacen eco en el tiempo}

[...] el conjunto de avances tecnológicos que nos proporcionan la informática, las telecomunicaciones y las tecnologías audiovisuales, que comprenden los desarrollos relacionados con los ordenadores, Internet, la telefonía, los "mass media", las aplicaciones multimedia y la realidad virtual. Estas tecnologías básicamente nos proporcionan información, herramientas para su proceso y canales de comunicación (MARQUÉS, 2001: 261).

La relación entre las TIC y el proceso de enseñanza-aprendizaje de lenguas

\footnotetext{
${ }^{8}$ Original: [...] o aluno já aprendeu usos da linguagem com os quais pode não ter se familiarizado em casa [...], tendo também interiorizado sua natureza sociointeracional [...] e começado a construir conhecimento de natureza metalinguística nas aulas de língua materna. [...] Isso lhe possibilita pensar, falar, ler e escrever sobre sua própria língua. Enfim, o aluno já sabe muito sobre sua língua materna e sobre como usá-la [...].
} 
existe de forma consistente hace varios años.

Podemos considerar que el uso metodológico de las TIC para fines educativos tuvo su inicio en la década de 40, época de la Segunda Guerra Mundial, más específicamente en los Estados Unidos, cuando se hicieron las primeras referencias específicas a la tecnología en el proceso de enseñanza-aprendizaje de idiomas, en cursos elaborados para el ejército de aquel país.

La primera vez que las TIC, o Nuevas Tecnologías, pasaron a figurar en un programa de formación universitaria fue en 1946, por medio de los cursos de Educación Audiovisual de la universidad de Indiana.

En la secuencia cronológica, percibimos que, conforme avanzaban los descubrimientos y mejoras tecnológicas, la utilización de las TIC en las clases de LE se hacía cada vez más variada y frecuente.

En la década de 40, con el advenimiento de los métodos audiolinguales, las TIC más recurrentes en las clases pasaron a ser el retroproyector, el grabador, el radio casete y el tocadiscos.

Con el surgimiento y la masificación del televisor, aparecieron los métodos audiovisuales, y hasta la década de 60 se agregó a las clases de enseñanza de LE esa tecnología y otras, como el proyector de cine y el video, lo que hizo con que surgiesen y creasen fuerza los llamados laboratorios de idiomas.

Por lo que percibimos, durante cerca de 30 años, la enseñanza de idiomas se pautó en enfoques metodológicos que utilizaron recursos tecnológicos variados; no obstante, a partir del surgimiento del ordenador y, más específicamente de la Internet, una verdadera revolución en las TIC aconteció, influenciando la Sociedad en general, y, por consiguiente, todos los procesos de enseñanza-aprendizaje.

De este modo, a partir de la década de 70, se incorporaron al proceso el ordenador, y, en los años 80, con el desarrollo de esa herramienta, el uso de los $P C$ multimedia, $C D s$, videos interactivos, $C D$-Roms, entre otros. El desarrollo no paró; por el contrario, amplió sus horizontes, y, a partir de la década de 90, con el advenimiento y la concretización de la Internet, se incorporaron al proceso de enseñanza-aprendizaje de lenguas variadas aplicaciones de esos avances como la propia web, la televisión a cable o vía satélite, el uso de $D V D$-Roms, $D V D$ s, pantallas y pizarras digitales interactivas, $P D A$ s, como los ordenadores portátiles y palms entre muchos otros, lo que amplió y está ampliando el campo de recursos tecnológicos disponibles a los profesores, y potencializando su papel como motivadores e incentivadores en el proceso de 
enseñanza-aprendizaje.

La inclusión de esas y otras TIC al proceso puede justificarse por las ventajas y beneficios que traen para las actividades humanas, incluyendo aquéllos específicamente educacionales, entre los cuales destacamos la facilitación del acceso a la información, por medio de canales de comunicación mediatos e inmediatos, la provisión de diferentes instrumentos para el almacenamiento y procesamiento de variadas cantidades y fuentes de datos, la homogeneización de códigos empleados para el registro de informaciones, la automatización de tareas, a partir de la programación de las actividades, y la interactividad, por medio de los ordenadores, la Internet, los juegos y materiales formativos multimedia.

Todos esos recursos funcionan como potencializadores cognitivos, pues activan y desarrollan nuestras capacidades mentales, permitiendo que sean buscadas y desarrolladas nuevas maneras de pensar sobre la información y la comunicación, y, por analogía, el proceso de enseñanza-aprendizaje de lenguas.

Por lo expuesto, percibimos que las TIC acompañan todos los avances en el proceso de enseñanza-aprendizaje, desarrollándose, modificándose y avanzando conforme a las necesidades impuestas por los cambios teórico-metodológicos, hecho que demanda una constante actualización institucional, tecnológica y, principalmente, docente.

Sin embargo, percibimos que no siempre esa actualización ocurre, y eso por variados motivos, los cuales alcanzan a las múltiples instancias relacionadas al proceso de enseñanza-aprendizaje. Y lo que más nos llama la atención es que todavía ocurren muchas prácticas metodológicas en la clase consideradas ya ultrapasadas, resumiéndose, muchas veces, solamente a la exposición.

Sobre esa temática, traemos a nuestra arena dialógica algunas otras voces, a seguir.

\section{Voces que hacen consideraciones sobre la clase expositiva}

La clase expositiva es una práctica muy común desde hace mucho tiempo, en todo el mundo, y está presente en la educación brasileña desde la época de los jesuitas. Algunos estudios apuntan que es la más utilizada por profesores y preferida por estudiantes de los niveles Básico y Superior (LOPES, 1993). A pesar de las críticas y fallas comúnmente apuntadas, siempre ha estado presente en las prácticas pedagógicas y 
en el proceso de enseñanza-aprendizaje.

Según Gil (2009: 68), “[...] en muchas carreras universitarias, se utiliza casi de forma exclusiva y son bastantes los profesores que identifican enseñanza con exposición."9

Algunas de las críticas hechas a esa práctica están basadas en la concepción de que el profesor es visto como el centro del proceso, de acuerdo a las palabras de Godoy (2003: 73) “[...] un tiempo de enseñanza ocupado enteramente o principalmente por la exposición de un ponente. Los estudiantes pueden tener la oportunidad de preguntar o de participar en una pequeña discusión, sin embargo en general sólo oyen y toman nota." 10

Esa práctica de enseñanza es denominada de modelo clásico de exposición (GIL, op. cit.). Se condensa la idea de que es posible transmitir conocimiento por medio de explicaciones orales; el profesor es el detentor del conocimiento, el orador; no se lleva en cuenta el conocimiento previo del alumno ni su experiencia de vida, y, en la mayoría de las veces, no se consigue atender individualmente a los discentes por falta de tiempo y de aproximación con ellos. No obstante, a pesar de las críticas a la exposición en clase, existen voces teóricas que afirman que esa práctica puede tener éxito, desde que complementada por el uso de recursos tecnológicos (GODOY, op. cit.). Según esas voces, cuando bien elaboradas y apoyadas por esos recursos, las clases expositivas suelen despertar la atención de los alumnos de manera significativa.

\section{VOCES DE NUESTRAS INVESTIGACIONES: BREVES RELATOS DE EXPERIENCIA}

La construcción de una investigación académica o científica normalmente parte de la observación y constatación de un problema, lo que genera una inquietud y una consecuente motivación, llevándonos a tejer hipótesis, con el objetivo de intentar entender las causas de ese problema y posteriormente apuntar caminos o alternativas.

La inquietud que llevó a la realización del trabajo de investigación en el Estado de Amazonas es oriunda de los estudios académicos de Graduación y Especialización de

\footnotetext{
${ }^{9}$ Original: “[...] em muitos cursos universitários, é utilizada quase de forma exclusiva e são em grande número os professores que identificam ensino com exposição."

${ }^{10}$ Original: "[...] um tempo de ensino ocupado inteiramente ou principalmente pela exposição de um conferencista. Os estudantes podem ter a oportunidade de perguntar ou de participar numa pequena discussão, mas em geral não fazem mais que ouvir e tomar apontamentos."
} 
uno de los autores de este artículo.

En aquellos momentos, al asistir a las clases, constató que muchos profesores utilizaban casi que exclusivamente el libro, el rotulador, la pizarra, y la voz, o sea, la exposición era la única forma de transmitir conocimiento, era la práctica dominante en el proceso de enseñanza-aprendizaje. Como eso le llamó la atención, pasó a dedicarse a esa temática y tejió la hipótesis de que alumnos de Secundaria tendrían preferencia por clases con dinámicas de grupo. Sin embargo, para su sorpresa constató que la preferencia de los alumnos era por clases expositivas. A partir de entonces, continuó de forma intensa a investigar la temática de la clase expositiva.

En octubre de 2009, dictando clases en el Nivel Superior, en una institución federal de enseñanza, notó que, al utilizar recursos tecnológicos variados, los alumnos asimilaban con menos dificultad los asuntos en estudio, lo que ellos confirmaron en diálogos sobre la temática en sala de clase.

Paralelamente, en sus estudios de Especialización lato sensu, se dedicó a la misma temática, con vistas a encontrar soluciones a cuestionamientos sin respuestas, entre ellos, saber los motivos por los cuales la exposición aún era la práctica más común en las clases, y si había información por parte de los docentes sobre la posibilidad de aliarse a la exposición el uso de las TIC en clase.

Durante esa caminata, hizo una nueva investigación, con la aplicación de un cuestionario $^{11}$, con el objeto de colectar datos de informantes, ya que se trata de un instrumento de investigación que tiene el objetivo de reunir informaciones sobre las percepciones, creencias y opiniones de individuos sobre objetos, personas y eventos presentes en su medio (MOURA; FERREIRA, 2005). Uno de los objetivos específicos era verificar las ventajas y limitaciones de la clase expositiva según la óptica de sus investigados.

Deseaba, asimismo, entender, entre otros puntos, el porqué del predominio de las clases expositivas tradicionales también en la Enseñanza Superior, considerando que los docentes las usan a menudo.

Los informantes ${ }^{12}$ eran profesores del Curso de Letras/"Língua Espanhola”, algunos de instituciones públicas y otros de instituciones privadas. Su edad variaba entre los 32 y 45 años, dos del sexo masculino y tres del femenino; uno de ellos había terminado la Maestría; tres eran Especialistas y uno era Graduado.

\footnotetext{
${ }^{11}$ Vide Apêndice 1: Cuestionario de investigación aplicado en el Norte de Brasil.

${ }^{12}$ Vide Galán (2010).
} 
Para realizar ese trabajo, levantó la hipótesis de que la clase expositiva es “[...] una de las modalidades de enseñanza más utilizadas por el profesor universitario en función de la propia estructura y carencia de recursos humanos y materiales que caracterizan la educación en la universidad en nuestro país (GODOY, op. cit.: 75)."13

Sin embargo, al final de las investigaciones, esa hipótesis no fue totalmente confirmada, ya que por lo verificado las instituciones en estudio disponían de recursos/TIC adecuados como, por ejemplo, proyectores de imágenes, computadores, televisores, radios casetes, aparatos de $D V D$, cámaras fotográficas, entre otros, $\mathrm{y}$, además, acceso, aunque con restricciones, a la Internet; todos disponibles para uso docente.

El principal motivo apuntado para el predominio de la clase expositiva sin que se usaran recursos de las TIC fue el factor tiempo. Cuando se les preguntó sobre la preferencia por prácticas para enriquecer una clase expositiva, la afirmación de que eran realizadas actividades con el apoyo de las TIC fue solamente sustentada por la minoría de los informantes. Lo que quedó claro fue que aún disponiendo de recursos adecuados no los utilizaban en sus clases a menudo, pues preferían la exposición. El motivo para esa actitud no fue conocido en aquélla investigación y una cuestión sobre esa temática se planteó: ¿Por qué profesores y educadores, actuantes en la Enseñanza Superior, no utilizan las TIC disponibles, prefiriendo la exposición?

El factor tiempo, y la falta de formación y de información son algunos ítems que pueden responder a esa cuestión.

Siguiendo la línea del discurso hasta el momento construido, afirmamos, con base en las investigaciones arriba detalladas, realizadas en el Estado de Río de Janeiro, que también se verificó la existencia de una laguna en la formación de los docentes que participaron como informantes de dicho trabajo ${ }^{14}$ en lo que se refiere al uso de las TIC en sus clases.

En aquel momento, se investigaba si los profesores que trabajaban en la enseñanza de Lengua Portuguesa ${ }^{15}$ y de E/LE tenían la costumbre de usar las TIC en sus clases. Para tanto, se usó un cuestionario de investigación ${ }^{16}$ y entrevistas realizadas por medio de técnicas de introspección.

\footnotetext{
13 Original: "[...] uma das modalidades de ensino mais utilizadas pelo professor universitário em função da própria estrutura e carência de recursos humanos e materiais que caracterizam a educação de terceiro grau no nosso país."

${ }^{14}$ Vide Teixeira (2010).

${ }^{15}$ En adelante: LP.

${ }^{16}$ Vide Apéndice 2: Cuestionario de investigación aplicado en el Sureste de Brasil.
} 
Según Tavares (1993: 37), "Introspección es el término que se usa en psicología y en lingüística aplicada para referirse genéricamente a distintos métodos de investigación de procesos mentales." ${ }^{\prime 17}$ Considerando que existen diversos métodos introspectivos de investigación, defendidos por diferentes estudiosos como Faerch y Kasper (1983), Cohen (1987), Ericsson y Simon (1987), entre otros, en este trabajo, se adoptó una clasificación sencilla, utilizada por Corrêa y Neiva, en artículo publicado en el año de 2000.

Los métodos introspectivos pueden clasificarse en dos tipos básicos: la introspección del procesamiento corriente y la retrospección, subdivididos en técnicas de investigación diferentes definidas a seguir.

La introspección del procesamiento corriente se subdivide en dos técnicas, la de 'pensar alto', a partir de la cual se realiza, por parte del individuo informante, un análisis de cada información accedida para la solución de un problema encontrado durante el proceso de lectura o de traducción; y la técnica de 'hablar alto', a partir de la cual el sujeto informante verbaliza oralmente su pensamiento corriente durante el proceso, sin que exista cualquier tipo de análisis.

Otro tipo de método introspectivo, llamado de retrospección, se utiliza para que el sujeto informante piense y se recuerde de las informaciones y pensamientos accedidos y ocurridos durante el proceso de lectura o de traducción. También se puede subdividir en dos técnicas distintas: la de 'retrospección inmediata', y la de 'retrospección retardada'.

Según Corrêa y Neiva (op. cit.), en la retrospección inmediata el relato hecho por el individuo informante sobre el proceso deberá ocurrir en hasta, lo máximo, treinta minutos después de terminar la tarea, mientras que en la retrospección retardada el relato ocurrirá a partir de un intervalo de treinta minutos del término de la tarea de lectura o de traducción.

En las entrevistas realizadas en ese trabajo se aplicaron los métodos introspectivos de 'pensar alto' y de 'hablar alto'. Cabe aquí resaltar que tanto el uso de cuestionarios como el uso de entrevistas configuran estrategias pertinentes a la investigación descriptiva, de acuerdo con las consideraciones teóricas de Cervo y Bervian (1983).

Los informantes fueron diez profesores de LP y 10 profesores de E/LE, todos

\footnotetext{
${ }^{17}$ Original: "Introspecção é o termo padrão usado em psicologia e em linguística aplicada para referir-se genericamente a diferentes métodos de investigação de processos mentais."
} 
residentes en ciudades fluminenses, Graduados en Letras, con Licenciatura Plena para la enseñanza de la LP y/o de E/LE.

Después de las investigaciones, se notó que cerca de la mitad de los docentes que trabajaban en la enseñanza de E/LE y la mayoría de los profesores que trabajaban en la enseñanza de LP no tenían la costumbre de usar las TIC en sus clases, por diferentes motivos, entre los cuales, los más recurrentes fueron la falta de conocimiento sobre las TIC y la falta de tiempo. Algunos incluso afirmaron desconocer esa terminología y otros que no sabían manosear tales recursos. El factor tiempo pareció ser el principal responsable por las barreras para el uso de las TIC, tanto en los trabajos con LP como en los trabajos con E/LE.

La gran mayoría de los profesores informantes afirmó que trabajan en más de una escuela y en más de una ciudad, perdiendo, así, una considerable parcela de tiempo con desplazamientos y adaptaciones a las realidades de las diferentes comunidades escolares. Tiempo precioso que podría aprovecharse para otras actividades, pues sin tiempo, los docentes no tenían como participar en cursos o eventos de formación, incluyendo aquéllos que versan sobre la temática de las TIC, llevando desinformación o falta de información sobre las potencialidades de esos recursos en clase.

Las TIC, cuando usadas, se trabajaban solamente cuando había tiempo sobrando en el planeamiento didáctico o en el desarrollo lectivo de las clases.

Quedó claro, así, que la falta de tiempo puede llevar a la falta de formación y a la consecuente falta de información docente de una forma general, incluyendo también la temática de las TIC en clase.

Además del factor tiempo, otros problemas aparecieron, como la falta de inversión o estímulo en la formación docente, sea por parte de las instituciones de enseñanza, sea por parte de las instancias gubernamentales.

A pesar de todos los problemas levantados hasta aquí, los cuales afectan al docente en su formación y actuación, un hecho verificado que merece destaque es la cuestión de que los profesores que trabajan con la enseñanza de LE demostraron poseer más conocimiento en lo que se refiere a la temática del uso de las TIC en clase e interesarse más por esa cuestión que los docentes que trabajan con la LP. Ese hecho, verificado en la realidad del Sureste brasileño, por analogía, podría también verificarse en otras Regiones de nuestro país, pues se sabe que la enseñanza de LP, a pesar de variadas sugerencias y esfuerzos para que se adopten innovaciones metodológicas y tecnológicas en clase, aún se pauta en abordajes mucho más tradicionales que las 
utilizadas en el proceso de enseñanza-aprendizaje de LE.

\section{ENSEÑANZA DE LENGUAS EN BRASIL: UN PARALELO SOBRE EL USO DE LAS TIC EN LAS REGIONES NORTE Y SUDESTE}

A partir de la arena de voces construida en este trabajo, percibimos que la enseñanza de lenguas en el Norte y en el Sureste del Brasil refleja una realidad que se observa hace años en el país entero: un proceso que todavía se pauta en modelos o abordajes teóricos bastante tradicionales. Tratándose de la enseñanza de LP, Lengua Materna de los brasileños, ese proceso demuestra estar más dependiente de abordajes impregnados del tradicionalismo, pues los profesores, aunque posean recursos tecnológicos y mediáticos a su disposición, prefieren la práctica de la exposición en clase.

Según los estudios desarrollados, percibimos, asimismo, que esa preferencia no se agota solamente en el ámbito docente, pues los alumnos también prefieren las clases expositivas, o al menos esperan que esa sea la práctica más recurrente para aprender una lengua, pues ya están acostumbrados a ella, en vista de que sus padres y abuelos aprendieron también de la misma forma.

En una época en que la información está disponible con sólo un clic, en que las TIC se desarrollan cada vez más rápido, trayendo nuevas posibilidades y potencializando los recursos didácticos en clase. ¿Qué es lo que provoca, entonces, que profesores y alumnos tengan esa postura frente a las prácticas en el proceso de enseñanza-aprendizaje de lenguas?

La respuesta a esta cuestión puede llevar a nuevas investigaciones y constataciones, en base a la realidad de esas y de otras Regiones brasileñas, a fin de componer un cuadro más completo sobre la temática. No obstante, podemos afirmar, en base a las informaciones que ya hemos colectado y analizado, que el factor tiempo es una de las grandes barreras que deben transponer los docentes para que el uso de las TIC pueda transformarse en una constante en las clases de lenguas, principalmente tratándose de la LP. Y, como una bola de nieve, otros problemas son generados como consecuencia de la falta de tiempo, abarcando cuestiones como la formación docente continuada, tan defendida y difundida por los medios de comunicación y por las instancias gubernamentales. Pero, no basta sólo defender y divulgar. Hay que colocar en práctica. Por eso, proponemos otras cuestiones: ¿Será que las políticas de incentivo y apoyo a la formación continuada docente realmente ocurren de forma efectiva y eficaz? 
¿Qué es lo que provoca, entonces, que cada vez más docentes trabajen en más de una escuela, muchas veces en ciudades diferentes? Son innúmeras las variables que pueden influir en las posibles respuestas a esas preguntas. Por eso, dejamos el hilo de esa tejedura para que los lectores, futuros investigadores, puedan contribuir con su(s) $\operatorname{voz}($ ces) en la formación intermitente de ese discurso, en la seguridad de que esa arena de voces no se agota aquí, en este momento, en este artículo.

\section{REFERÊNCIAS}

BRASIL (2005). Presidência da República. Casa Civil. Subchefia para Assuntos Jurídicos. Lei 11.161/2005. Disponible en http://www.smec.salvador.ba.gov.br/site/documentos/espaco-virtual/espacolegislacao/EDUCACIONAL/NACIONAL/lei\%20n\%C2\%BA\%2011161$2005 \% 20$ ensino $\% 20$ da\%20lingua\%20espanhola.pdf. Accesado en 23/05/2009, a las $16 \mathrm{~h}$.

BRASIL (1998). Secretaria de Educação Fundamental. Parâmetros curriculares nacionais: terceiro e quarto ciclos do ensino fundamental: introdução aos parâmetros curriculares nacionais. Brasília: MEC/SEF.

BRASIL (1999). Secretaria de Educação Média e Tecnológica. Parâmetros curriculares nacionais: ensino médio. Brasília: MEC/SEMT.

CERVO, A. L.; BERVIAN, P. A. (1983). Metodologia científica: para uso dos estudantes universitários. 3. ed. São Paulo: McGraw Hill do Brasil.

COHEN, A. D. (1987). Using verbal reports in research on language learning. In: FAERCH, K.; KASPER, G. (orgs.) Introspection in second language research. Clevedon, Philadelphia: Multilingual Matters.

CORRÊA, A. M. da S.; NEIVA, A. M. S. (2000). Estratégias e problemas do tradutor aprendiz: uma visão introspectiva do processo tradutório. In: MONTEIRO, M. J. P. (org.) Práticas discursivas: instituição, tradução e literatura. Rio de Janeiro: UFRJ, Faculdade de Letras.

ERICSSON, K. A.; SIMON, H. A. (1987). Verbal reports on thinking. In: FAERCH, K.; KASPER, G. (orgs.) Introspection in second language research. Clevedon, Philadelphia: Multilingual Matters.

FÁVERO, L. L. (2003). Paródia e dialogismo. In: BARROS, D. L. P. de; FIORIN, J. L. (orgs.). Dialogismo, polifonia e intertextualidade. 2. ed. 1. reimpr. São Paulo: EdUSP. (Ensaios de Cultura, 07)

FAERCH, K.; KASPER, G. (1983) Plans and strategies in foreign language communication. In: (orgs.). Strategies in interlanguage communication. 
London: Longman.

GALÁN, S. J. (2010). Aula de espanhol na universidade: uma reflexão sobre a aula expositiva num curso de letras língua espanhola em Manaus. Manaus. Artigo (Lato Sensu) apresentado ao Curso de Especialização em Metodologia do Ensino Superior da Escola Superior Batista do Amazonas.

GIL, A. C. (2009). Metodologia do Ensino Superior. 4. ed. São Paulo: Atlas.

GODOY, A. S. (2003). Revendo a Aula Expositiva. In: MOREIRA, Daniel A. (org.). Didática do Ensino Superior. São Paulo: Pioneira.

LOPES, A. O. (1993). Aula Expositiva: superando o tradicional. In: VEIGA, Ilma P. A. (org.) Técnicas de Ensino: por que não? 2. ed. São Paulo: Editora Papirus.

LOPES, L. P. M. Oficina de lingüística aplicada. 3. reimp. Rio de Janeiro: Mercado das Letras, 2001.

MÁRQUES, P (2001). Las TIC y sus aportaciones a la sociedad. Disponível em http://dewey.uab.es/pmarques/tic.htm, Accesado en 10/01/2009, a las 10h.

MOURA, M. L. S. de; FERREIRA, M. C. (2005). Projetos de pesquisa: elaboração, redação e apresentação. Rio de Janeiro: EDUERJ.

TAVARES, K. C. do A. (1993). O uso da introspecção: da técnica de pesquisa para o ensino de leitura. Rio de Janeiro: UFRJ, Faculdade de Letras. 239 fl. mimeo. Dissertação de Mestrado em Letras Anglo-Germânicas.

TEIXEIRA, W. B. (2010). O uso das tecnologias da informação e da comunicação em aulas de língua materna e estrangeira. Rio de Janeiro. Monografia (Lato Sensu) apresentada ao Curso de Especialização em Educação Tecnológica do Centro Federal de Educação Tecnológica Celso Suckow da Fonseca.

\section{LOS AUTORES}

Santiago Javier Galán es Especialista en Metodología de la Enseñanza Superior por la Escola Superior Batista do Amazonas/AM y Graduado en Letras, con habilitación en la Lengua Española por la misma institución y en la Lengua Portuguesa por la Universidade Federal do Amazonas/AM. Actualmente es Profesor Auxiliar del Departamento de Lenguas y Literaturas Extranjeras de la UFAM. Sus investigaciones se direccionan a la enseñanza de lenguas y al uso de las Nuevas Tecnologías en el proceso de enseñanza-aprendizaje.

E-mail: santiagomanaus@yahoo.com.br

Wagner Barros Teixeira tiene la Maestría en Estudios Linguísticos Neolatinos por la Universidade Federal do Rio de Janeiro/RJ y es también Especialista en Educación Tecnológica por el Centro Federal de Educação Tecnológica Celso Suckow da Fonseca/RJ. Actualmente es alumno del Doctorado en el mismo programa de Pos Grado 
de su Maestría. Es Profesor Asistente del Departamento de Lenguas y Literaturas Extranjeras de la Universidade Federal do Amazonas y sus investigaciones se direccionan a la enseñanza de lenguas, el proceso de lectura, el discurso, y las Nuevas Tecnologías en la Educación.

E-mail: wagbarteixeira@ufam.edu.br

\section{APÉNDICES}

\section{Apéndice 1: Cuestionario de investigación aplicado en el Norte de Brasil}

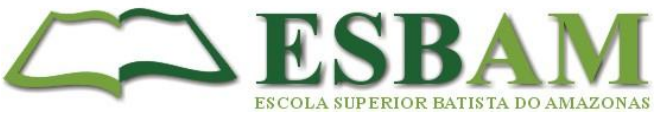

Cuestionario para elaboración de monografía:

$1^{\circ}$ iAlguna vez usted ha hecho un curso de español?

$\square \quad$ Sí

$\square \quad$ No

$\square \quad$ Sólo estudié en la escuela.

$2^{\circ}$ Dentro del estudio de la lengua española, ¿Cuál de los puntos abajo más se aproxima a su realidad?

$\square \quad$ Me gusta el español y lo asimilo con facilidad

$\square \quad$ Nunca me gustó el español

$\square \quad$ Me gusta el español, pero siempre tuve dificultad en aprenderlo con facilidad.

$3^{\circ}$ El trabajo en grupo, ¿facilita o dificulta su aprendizaje?

Facilita

$\square \quad$ Dificulta

$4^{\circ}$ ¿Cómo usted piensa que aprende mejor? Numere en orden de preferencia de 1 a 4 .

$\square \quad$ Por medio de números.

$\square \quad$ Con explicaciones orales.

$\square \quad$ Escuchando músicas y cantando.

$\square \quad$ Leyendo y escribiendo.

$\square \quad$ Hablando, escuchando y debatiendo.

$\square \quad$ Con dibujos coloridos y películas.

$\square \quad$ Bailando y dramatizando.

$\square \quad$ Solo y realizando tareas individuales.

$\square \quad$ En grupos por medio de dinámicas.

Questionário para elaboração de artigo 
1. Qual é a sua concepção sobre aula expositiva e dê sua opinião sobre sua utilização em sala de aula na universidade?

2. De 1 a 4, aponte em ordem de importância as vantagens de uso de uma aula expositiva tradicional.

Tempo: Em pouco tempo se transmite muitas informações e geralmente o conteúdo já está pronto.

Praticidade e Economia: Com poucos recursos didáticos pedagógicos disponíveis se consegue atingir a um grande número de alunos.

Os alunos estão mais habituados a aprender escutando do que lendo, pesquisando ou produzindo.

Outros:

3. Em sua opinião, numerando de 1 a 5 por ordem de importância, aponte as limitações de uma aula expositiva tradicional.

Não se considera de que alguns alunos não possuem conhecimento prévio sobre o tema da aula.

Não favorece o desenvolvimento de habilidades intelectuais mais complexas como a produção, análise, síntese e julgamento.

O docente não consegue atender a todos os alunos, considerando a particularidade de cada um, os quais têm pouca participação.

Cria o hábito de que os alunos estudem somente por suas anotações de classe.

Outros

4. Em ordem de preferência, sugira atividades ou técnicas que podem complementar ou enriquecer uma aula expositiva.

Atividades lúdicas

Dinâmicas de grupo

Seminários e debates

Painel integrado

Atividades com o uso de recursos tecnológicos

Aulas dialógicas

Leituras dinâmicas

Outros 


\section{Apéndice 2: Cuestionario de investigación aplicado en el Sureste de Brasil}

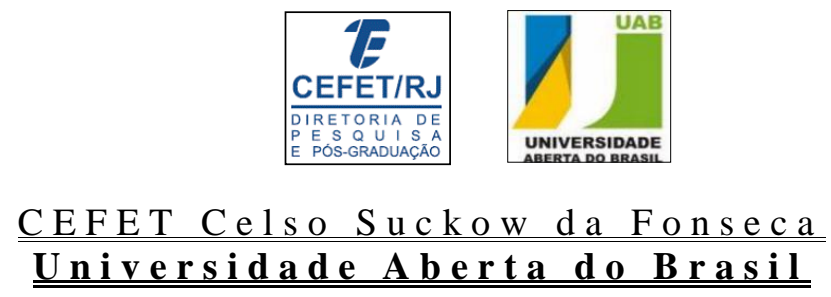

\section{QUESTIONÁRIO SOCIOLINGUÍSTICO}

1. Você costuma utilizar algum tipo de recurso em suas aulas de língua?

( ) $\operatorname{Sim} \quad($ ) Não

2. Quais recursos você utiliza?

( ) Quadro negro ou branco

) Mapas, figuras e desenhos variados

( ) K7 ou CD player

( ) TV e vídeo ou DVD player

( ) Computador e Internet

(

3. Você já participou de algum evento ou curso que promovesse a formação sobre as TIC?

( ) Sim, já participei antes, mas não tenho mais interesse em participar.

( ) Sim, já participei e ainda tenho interesse em participar de outros.

( ) Não, nunca participei, mas tenho interesse em participar.

( ) Não, nunca participei, e não tenho interesse em participar.

4. Se sua resposta foi negativa à questão de número 4 (quatro), justifique-a, a seguir:

( ) Não me interesso mais pelo tema.

( ) Não sei o que são as TIC.

( ) Não tenho tempo para participar de eventos ou cursos.

( ) Já conheço bastante sobre o tema, portanto, não é necessário que me interesse mais sobre o assunto.

( ) ) Outros motivos:

5. Você já recebeu alguma ajuda ou incentivo para participar de eventos ou cursos como os mencionados no item 3?

( ) Sim, de minha escola.

( ) Sim, da Secretaria de Educação.

( ) Sim, de minha família ou amigos.

( ) Sim, de agências de fomento à pesquisa.

( ) Não, nunca recebi.

6. Em quantas e quais cidades leciona?

( ) Em somente 01.

( ) Em duas.

( ) Em três.

( ) Em quatro ou mais.

$\mathrm{Na}(\mathrm{s})$

cidade(s) 
( ) Em apenas 01 .

( ) Em duas.

( ) Em três.

( ) Em quatro ou mais.

8. $\mathrm{Na}(\mathrm{s})$ escola(s) onde você trabalha, com que freqüência utiliza espaços como BIBLIOTECA, VIDEOTECA e SALA DE MULTIMIDIA em suas aulas?

( ) Sempre, em quase todas as aulas estão disponíveis.

( ) Às vezes, quando disponho de tempo sobrando em meu planejamento.

( ) Quase nunca, pois na maioria das vezes não tenho tempo.

( ) Nunca, pois não dá tempo.

( ) Nunca, pois não sei utilizá-las.

( ) Não há BIBLIOTECA disponível em minha escola.

( ) Não há VIDEOTECA disponível em minha escola.

( ) Não há SALA DE MULTIMIDEA disponível em minha escola.

9. Quais são as maiores dificuldades que você encontra ao trabalhar com as TIC?

( ) O desinteresse dos alunos.

( ) A falta de oportunidade e apoio da instituição escolar.

( ) A ausência de conhecimentos teóricos sobre o assunto.

( ) A falta de material e/ou de infra-estrutura para apoiar o trabalho.

(

10. Qual a importância do uso das TIC nas aulas de idiomas?

Desde já, autorizo a reprodução e divulgação das informações contidas aqui, bem como nos protocolos verbais por mim realizados para fins de pesquisa acadêmica e científica.

Assinatura: I__ $/ 2010$. 